\title{
Effect of Fluid Dynamics and Device Mechanism on Biofluid Behaviour in Microchannel Systems: Modelling Biofluids in a Microchannel Biochip Separator
}

\author{
Xiangdong Xue ${ }^{1 *}$, Mayur K Patel ${ }^{1}$, Maïwenn Kersaudy-Kerhoas ${ }^{2}$, Chris Bailey ${ }^{1}$, Marc P.Y. Desmulliez ${ }^{2}$, David Topham ${ }^{3}$ \\ ${ }^{1}$ School of Computing and Mathematical Sciences, University of Greenwich, London SE10 9LS, UK \\ ${ }^{2}$ MIcroSystems Engineering Centre (MISEC), School of Engineering \& Physical Sciences \\ Heriot-Watt University, Edinburgh, EH14 4AS, UK \\ ${ }^{3}$ School of Engineering and Design, Brunel University, Uxbridge, Middlesex, UB8 3PH, UK \\ *X.Xue@gre.ac.uk
}

\begin{abstract}
Biofluid behaviour in microchannel systems is investigated in this paper through the modelling of a microfluidic biochip developed for the separation of blood plasma. Based on particular assumptions, the effects of some mechanical features of the microchannels on behaviour of the biofluid are explored. These include microchannel, constriction, bending channel, bifurcation as well as channel length ratio between the main and side channels. The key characteristics and effects of the microfluidic dynamics are discussed in terms of separation efficiency of the red blood cells with respect to the rest of the medium. The effects include the Fahraeus and Fahraeus-Lindqvist effects, the Zweifach-Fung bifurcation law, the cell-free layer phenomenon. The characteristics of the microfluid dynamics include the properties of the laminar flow as well as particle lateral or spinning trajectories. In this paper the fluid is modelled as a single-phase flow assuming either Newtonian or Non-Newtonian behaviours to investigate the effect of the viscosity on flow and separation efficiency. It is found that, for a flow rate controlled Newtonian flow system, viscosity and outlet pressure have little effect on velocity distribution. When the fluid is assumed to be Non-Newtonian more fluid is separated than observed in the Newtonian case, leading to reduction of the flow rate ratio between the main and side channels as well as the system pressure as a whole.
\end{abstract}

\section{Introduction}

Microfluidic packaging has been gaining increased attention in Microsystems. A typical application is in the healthcare field, where microfluidic devices containing microchannels have shown an increasing number of applications in the biological and clinical areas. Computational Fluid Dynamics (CFD) can provide valuable insights at the early stage of the design on the performance such devices as well as the impact that the packaging might have on operational performance.

An important use of microfluidic devices in biological applications is in blood separation. Due to the difference in density between blood cells and plasma, human blood is usually separated by centrifugation where inertial forces tend to play a dominant role [1]. This approach, not so effective in microsystem devices, has led to an interest in adopting new microscopic separation methods, in which viscous forces, shear strain rate, surface tension and the geometrical effects of microchannels play important roles.

Separation methods using fluid mechanical effects may be classified into two categories: micro filter devices [2-3] and microchannel devices [4-5]. Among the latter, channel constriction [6-7], bending channel [8-9] and bifurcated channels [10-11] have been explored. In this paper, a Tshaped microchannel design [12-13] is investigated using CFD where the aim of the design is to separate the biofluid (i.e. blood) via multiple bifurcations. The resulting device consists of a main channel and a series of perpendicularly positioned branch/side channels. When the flow rate ratio between the main and side channels reaches a critical level, plasma is separated into the side channels.

The main difference between micro- and macrofluids comes from the effect that particles/cells have on the flow fields. Containing a vast number of particles, a macro system is generally considered as a single phase continuum flow, whereas with channel size comparable to the particles, a microsystem has to take into account particle behaviour on the bulk flow of the fluid. Consequently, for fluids such as blood, the suspension of cells in plasma, and their interactions, will affect the overall behaviour of the microfluidic device.

There are generally two modelling approaches in studying particle (or cell) performance in a bulk flow. One is the discrete method, in which the individual cells or a collection (PSI cell) are modelled by the immersed boundary method (IBM) or the immersed finite element method (IFEM) and the interactions between cells and the bulk flow are explicitly represented [14-15]. The other approach is the representation of the bulk flow field as a single-phase fluid with bulk properties to represent the affects of the cells [5, 12]. Of the two approaches, the former is mainly used for investigating detailed behaviour of particles and the flow in local regions. The latter is used for describing the global performance of the mixture (particles and fluid) of the flow in the system or device. In this paper, effort is concentrated on the latter approach.

The important phenomena for biofluid behaviour in microchannels has been explained by several laws or effects, including Fahraeus effect and Fahraeus-Lindqvist effect [16], Zweifach-Fung bifurcation effect [17-18] and the so-called cell-free or liquid-skimming layer effect.

The Fahraeus effect and Fahraeus-Lindqvist effect are strongly correlated, relating to the effect of changing channel cross-sections, such as a constriction, on flow hematocrit and its viscosity. It was observed [19] that, in a channel of $20 \mu \mathrm{m}$ diameter (this is the channel size in this study), the viscosity is about $50 \%$ of its normal value. The existence of a cell-free layer close to the channel walls can be from both effects indicated above. For laminar flow, the cell-free layer is 
always located in the layers close to the wall where the liquid can be extracted by appropriately designed apertures or branches on the wall. The Zweifach-Fung effect concerns cell behaviour at these bifurcations, inferring that particles have a tendency to travel through the channels with higher flow rate ratio.

In microfluidic channel systems, mechanical (i.e. geometrical) details also play an important role on the behaviour of the flow field. For the separation of blood plasma specifically, two geometrical effects of importance are channel constriction and channel bending. When blood passes through a channel constriction, the cells tend to travel faster and concentrate at the centre of the channel which results in a higher concentration of plasma near the wall, where it can be collected or skimmed from. A bending channel makes use of the centrifugal force induced on the particles when passing through the curved section, thus ensuring a higher concentration of plasma in the central region of the channel, where it can be collected or skimmered from.

Diluted and high sheared biofluids generally behave like Newtonian flows, but biofluids with higher cell concentrations show non-Newtonian behaviour. The difference is addressed in the current paper through the comparison of modelling results obtained by both Newtonian and non-Newtonian flows for a blood separator design. The biochip is introduced and analysed. This is followed by the modelled phase using CFD to investigate the flow field as a function of separation performance. Finally, comparisons of Newtonian and non-Newtonian flows are presented and discussed.

\section{The Biochip and Fluid Dynamics}

\subsection{Device}

Figure 1 shows the first prototype of the whole system separation device, together with system level controls at the micro level. The components at the system level include a syringe (1 4) for pumping the biofluid sample (e.g. blood) into the inlet $(5)$, collection equipment $(11,12)$ of the separated fluid (e.g. plasma) at the outlets $(6,7)$ and concentrated fluid containing cells at the outlet (10), a counting equipment for examining cell concentration, and tubes and couplers between the micro and macro levels. The biochip (8), Figure 1(b), is the core part of the separator. Blood is pumped through the inlet at the top and flows downwards. After passing a constriction, the blood enters the main channel which contains a number of side branches where bifurcation occurs. Through these bifurcations, blood is separated into high concentrations of blood cells (main channel) and plasma (side channels).

In this example the size of the biochip is $7 \mathrm{~mm} \times 10 \mathrm{~mm}$. The width of the main channel is $100 \mathrm{~m}$ and the constriction and branch side channels are $25 \mu \mathrm{m}$ and $20 \mu \mathrm{m}$, respectively. The depth of all channels is $20 \mu \mathrm{m}$. Due to the size of the biochip and associated micro-channel dimensions a high aspect ratio of length to cross-sectional area is evident.

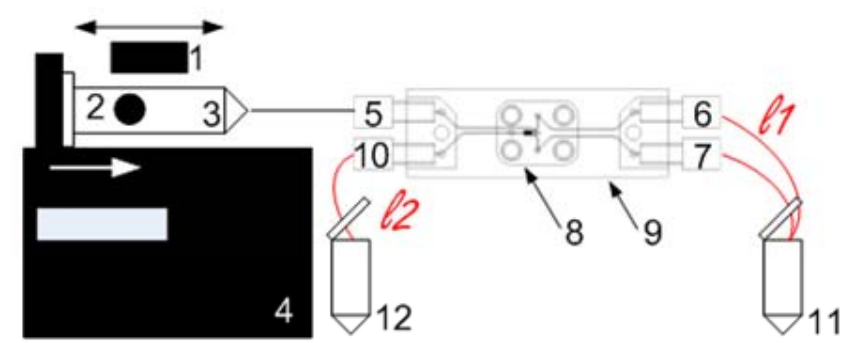

(a) the experimental setup

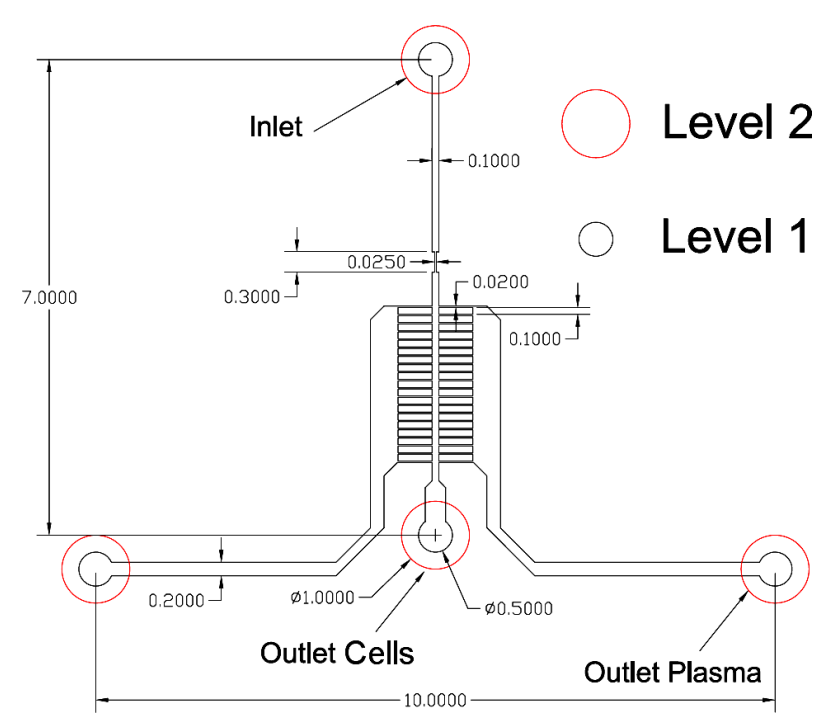

(b) the biochip in micro level, unit: $\mathrm{mm}$

Figure 1 Separation device [20]

\subsection{Device features and microfluidic dynamics}

\subsubsection{Microchannel}

Microchannels have high surface-to-volume ratio. Assuming a no-slip condition for the fluid at the channel walls, the flow velocity cannot be developed to a high level in this micro space. High surface-to-volume ratio also means the flow in microchannels suffers relative high resistive force at its boundary surface on the wall. As a result, a laminar flow with low velocity, high pressure, high shear stain rate and low Reynolds number is common in microchannels, in which viscous forces play an important role on the behaviour of the flow field. When the channel size is reduced to $300 \mu \mathrm{m}$ or less [16], the Fahraeus effect and Fahraeus-Lindqvist effects are also important. A cell-free layer is formed close to the wall, which provides a distinctive feature for separating plasma from the bulk blood flow.

Spinning is an important mode for moving a particle. Under parabolic flow distribution, the flow velocity at the channel centre and near wall regions are substantially different. When a cell/particle is located to the left/right of the centre line of the channel, it experiences different flow velocities and shear stresses on the two opposite sides parallel to the bulk flow. This results in different forces acting on the two sides which, in turn, induce spinning. This difference in velocity results in a pressure drop across the particle and 
drives the cell towards the high velocity region, i.e. the channel centre. Here the relevant lift forces are known as the Magnus force [21] and Saffman force [22]. Note that these lift forces are produced when a particle is located to the left/right of the centre line of the channel. When the particle crosses the channel central line, this effect is substantially weakened.

\subsubsection{Constriction}

A constriction has three functions in terms of blood plasma separation: focusing the blood flow from the central region of the connected channel section, accelerating the flow and increasing the velocity difference between cells and plasma. The purpose of the first function is to concentrate the cells towards the channel centre. With the size effect, more plasma is being diffused to channel periphery region than blood cells.

The velocity changes when a flow passes a constriction can be explained via the mass conservation law, i.e. the continuity equation, as follow:

$$
\frac{\partial \rho}{\partial t}+\operatorname{div}(\rho v)=0
$$

where $\rho$ is the density of the flow and $v$ the velocity. For a well-developed incompressible flow, $\partial \rho / \partial t=0$. Ignoring the small density change when the flow passes the constriction and expressing Eq. (1) in integral form, we get

$$
\int_{\text {chan }} V_{\text {chan }} d A_{\text {chan }}=\int_{\text {cons }} V_{\text {cons }} d A_{\text {cons }}
$$

where $V$ denotes flow velocity, $A$ the area of channel crosssection; the suffixes chan and cons represent the main channel and constriction. Expressed with average forms, Eq. (2) becomes

$$
\frac{V_{\text {cons }}}{V_{\text {chan }}}=\frac{A_{\text {chan }}}{A_{\text {cons }}}
$$

As the cross section of the constriction is $1 / 4$ of that of the main channel, the velocity in the constriction increases by a factor of four where the average velocity can be around $200 \mathrm{~mm} / \mathrm{s}$.

The change in relative velocities between blood cells and plasma with the constriction can be explained by the Fahraeus effect. The Fahraeus effect states that when blood of a given hematocrit (i.e. cell concentration), flows from a large reservoir into a tube of small diameter, the hematocrit in the tube decreases as the tube diameter decreases [16]. As the hematocrit at both sides of the constriction is the same and the thickness of the cell-free layer is relatively constant, a lower hematocrit level in the constriction means that the blood cells move faster than the plasma. As a result, blood cells are more likely to stay at the channel central region and the hydrodynamic effect becomes more effective.

\subsubsection{Bifurcation}

Zweifach-Fung bifurcation law is the most important effect for the current device. Based on this empirical law, the separation efficiency is a function of flow rate ratio between the main and side channels. It was observed [12] that, for a cell to channel diameter ratio of the order of 1 , when the flow rate ratio reaches $6: 1-8: 1$, nearly all cells will travel through the channel with the higher flow rate, leaving almost no cells travelling into the slower flow rate channel.

The effect of bending channels is also effective at bifurcations. As the side channels are perpendicularly connected with the main channel and the fluid flows into a side channel from the main channel, the fluid suffers a centrifugal force. As the density of blood cells is higher than plasma, under the inertia effect, blood cells have a tendency to move away from the bending flow to return to the flow in the main channel. The centrifugal force is directly proportional to the square of the flow velocity.

\subsubsection{Channel size and outlet locations}

The cross-section of the main channel is larger than the side channels. The bifurcation region is much closer to the outlet of the main channel than the outlets of side channels. The purpose of the above design is to produce a high flow rate ratio between the main and side channels for separation purpose.

The relationship of channel resistance to channel length, cross area and viscosity is expressed by Poiseuille's law, which states that, for the laminar incompressible fluid, the resistance of a channel is directly proportional to channel length and flow viscosity and inversely proportional to the fourth power of diameter (i.e. the square of the area) of a circular channel cross-section, expressed as follow.

$$
R=C \cdot \frac{L \mu}{A^{2}}
$$

where $R$ denotes channel resistance, $L$ the channel length, $A$ channel cross-section area, $\mu$ flow viscosity and $\mathrm{C}$ a coefficient. From Eq. (4), the ratio of channel resistance between the main and side channels can be expressed as

$$
\frac{R_{m}}{R_{s}}=C^{\prime} \cdot \frac{L_{m} \mu_{m}}{L_{s} \mu_{s}}\left(\frac{A_{s}}{A_{m}}\right)^{2}
$$

in which suffixes $m$ and $s$ denote the main and side channels, respectively.

Eq. (5) shows that the ratios of channel lengths and crosssections between the main and side channels determine the channel resistance ratio, i.e. the flow rate ratio. It also shows that viscosity variation of the fluid has a strong effect on flow field. Hence, non-Newtonian behaviour of the fluid should be taken into account in modelling biofluid flows.

\section{Modelling}

\subsection{Newtonian and Non-Newtonian Flows}

In a Newtonian flow, the rate of shear stress and strain, i.e. viscosity, is constant. When human blood is considered as a Newtonian fluid, the bulk flow is modelled with a constant viscosity of $0.0035 \mathrm{~Pa} . \mathrm{s}$ and a density of $1060 \mathrm{~kg} / \mathrm{m}^{3}$. To investigate the effect of viscosity on flow field, horse blood is also modelled. For horse blood (Newtonian), the flow is modelled with a viscosity of 0.0047Pa.s [23] and a density of $1060 \mathrm{~kg} / \mathrm{m}^{3}$ [24].

When human blood is considered Non-Newtonian, two fluid models are implemented to the main channel and side channels, respectively. The fluid in the side channels and subsequent outflow region is modelled as plasma with the 
constant viscosity of $0.0015 \mathrm{~Pa} . \mathrm{s}$ and a density of $1025 \mathrm{~kg} / \mathrm{m}^{3}$. The flow in the main channel is modelled with a shear rate dependent non-Newtonian flow. The Carreau-Yasuda model is used for modelling the shear-thinning behaviour of the fluid, shown as follow:

$$
\mu(\dot{\gamma})=\mu_{\infty}+\frac{\mu_{0}-\mu_{\infty}}{\left(1+(\lambda \dot{\gamma})^{b}\right)^{a}}
$$

where $\dot{\gamma}$ is the shear rate, $\mu_{\infty}$ and $\mu_{0}$ are the infinite shear viscosity and the zero shear viscosity, respectively; $\lambda$, $a$ and $b$ are constants. Parameter values are as follows $[25,26]: \mu_{\infty}=$ $0.0035 \mathrm{~Pa} . \mathrm{s}, \mu_{0}=0.16 \mathrm{~Pa} . \mathrm{s}, \lambda=8.2 \mathrm{~s}, \mathrm{a}=1.23$ and $\mathrm{b}=0.64$. The fluid density is taken as $\rho=1060 \mathrm{~kg} / \mathrm{m}^{3}$. The Carreau-Yasuda model shows that non-Newtonian blood flow is a shear-thin flow.

\subsection{Computational model}

The three-dimensional Computational Fluid Dynamics (CFD) finite-volume package Ansys-CFX (CFX5) is used to perform the analysis. A thin-layer 3D model, in effect $2 \mathrm{D}$, to save computer resources, is developed. Figure 2 shows the computational model together with the mesh at the first upstream bifurcation and the inlet side of the constriction. A three-layer fine mesh is constructed in the region close to the wall to represent the flow performance in the boundary layer. In total 605,243 mesh elements and 654,954 nodes are contained in the model. The coordinate origin is set at the left hand end of the last downstream side channel, as shown in the graphs below.

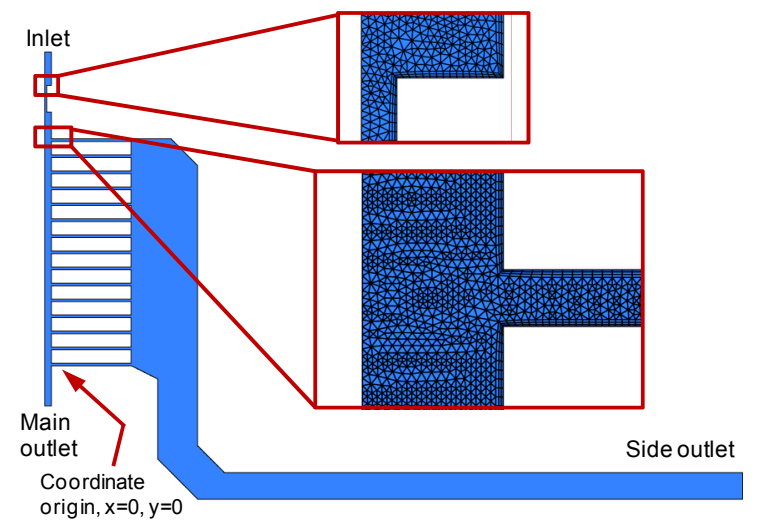

Figure 2 Computational model and the mesh in the first upstream bifurcation

Due to the symmetric nature of the device, and assuming that the flow is symmetric, only half of the device needs to be modelled. This consists of 15 side channels and one main channel as illustrated in Figure 2. For the boundary conditions, the inlet is a constant flow rate of $360 \mu \mathrm{L} / \mathrm{h}$ $(50 \mathrm{~mm} / \mathrm{s})$. Both boundary conditions at the main and side outlets are set to $0 \mathrm{~Pa}$ pressure. For a well-developed incompressible laminar flow, the flow field in the biochip is only determined theoretically by the relative pressure between the inlet and outlets. Thus, the result obtained from $0 \mathrm{~Pa}$ outlet pressure is also suitable to other pressure levels.

\section{Results and Comparison}

\subsection{Modelling biofluids using Newtonian flow}

Figure 3 shows the Reynolds number in the main and side channels at the first upstream and the last downstream bifurcations, respectively. With lower flow rate, the Reynolds number in the side channels is one order of amplitude lower than the main channel. Laminar flow character is evident by the low Reynolds numbers.

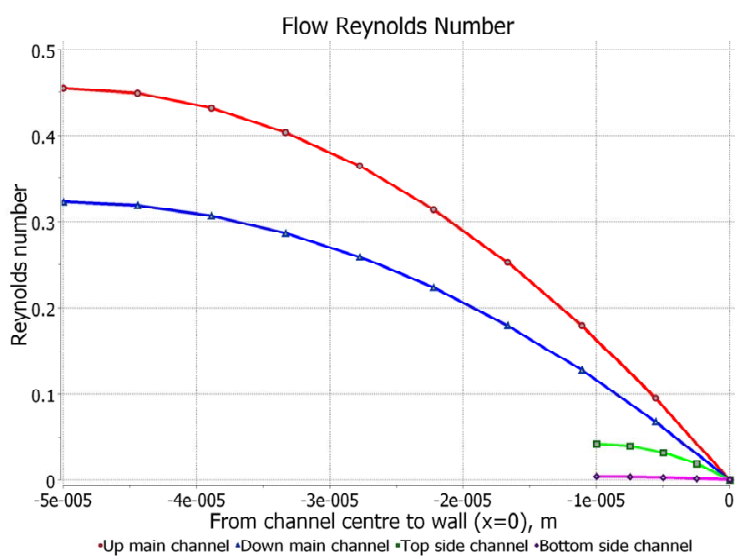

Figure 3 Reynolds number of channels

Figure 4 shows a close view of the 'creeping' flow pattern in the first upstream channel. The reduction of the fluid velocity from the centre of the main channel to the side channels is clearly visible. Only the fluid streamlines close to the wall of the main channel, where the cell-free layer is located, enters the side channels promoting separation.

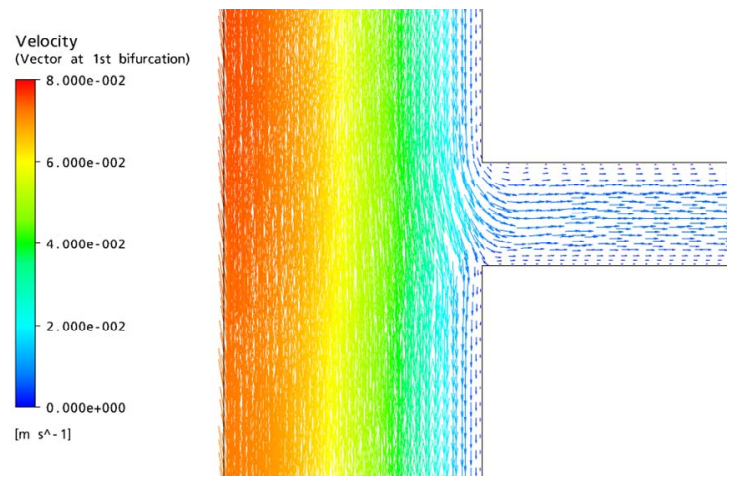

Figure 4 Velocity vector contour in the first upstream channel

Figures 5 through 7 show the velocity profile development through the microchannel biochip. Figure 5 shows the flow velocity profile within the constriction. The maximum velocity inside the constriction is about four times of that in the main channel. This difference in velocity is attributed to Eq. (3).

Figure 6 shows the velocity profile in the main channel. The parabolic profile of the velocity is closely related to a cross channel motion in the main channel, as discussed in section 2.2.1. The velocity distribution also indicates the difference of shear rate across the channel. The maximum 
shear strain rate appears on the boundary layer close to the wall.

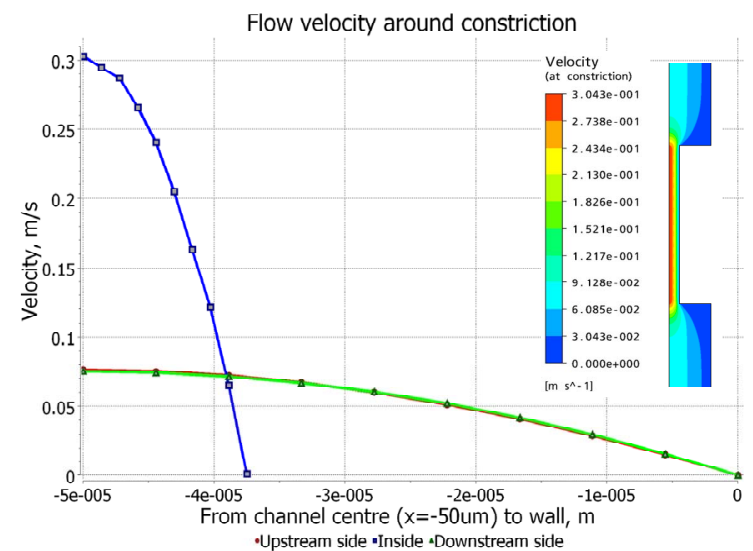

Figure 5 Flow velocity profile around constriction with velocity contour inserted

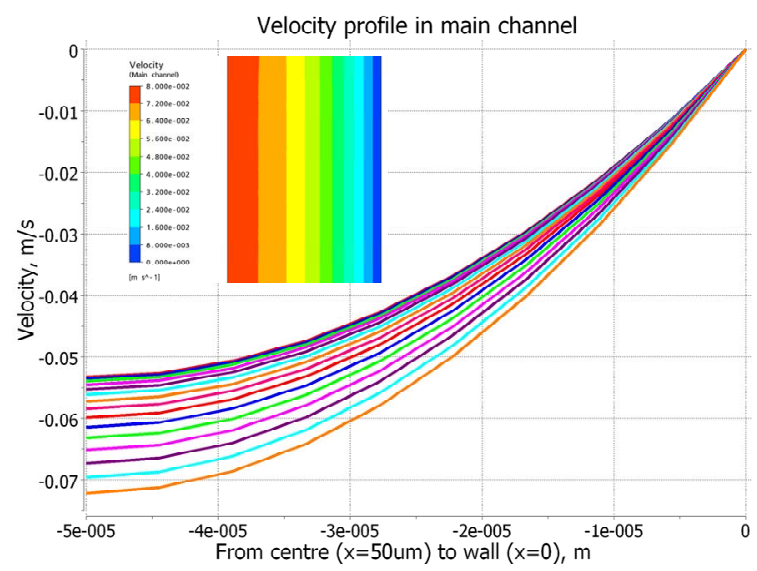

Figure 6 Flow velocity profile in the main channel with contour inserted

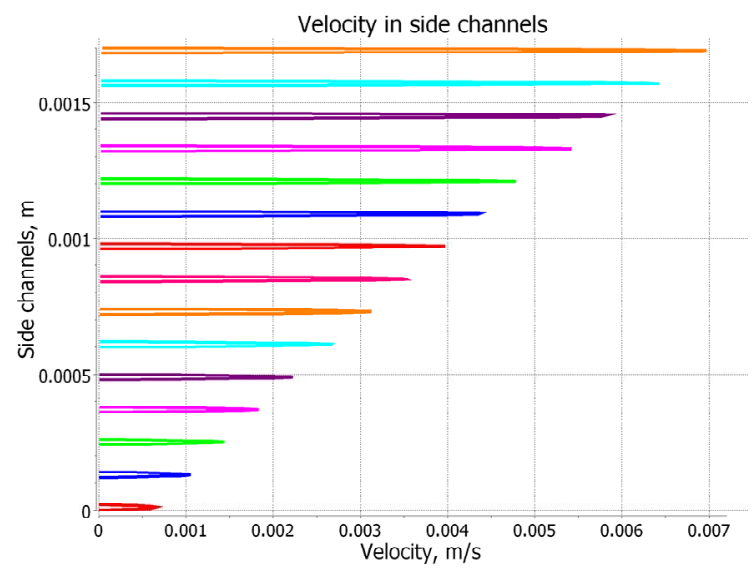

Figure 7 Flow velocity of side channels

Figure 7 shows the velocity of side channels. The velocities vary substantially upstream and downstream side of the channels, showing a linear reduction from upstream to downstream channels. This is because, in order to form high flow rate ratios between the main and side channels, the outlet of the main channel is designed to be closer to the bifurcation region as opposed to the outlet of side channels (see Figure $1)$.

Horse blood is modelled with slightly higher viscosity than human blood fluid. The modelling result shows that the velocity distribution of horse blood flow is nearly identical as the human blood flow case, i.e. Figures 3 to 7 . This implies that, for the flow rate controlled cases, the viscosity has little effect on the velocity distribution pattern. Combining the analysis in section 3.2 about the effect of the outlet pressure, we can conclude that, for a Newtonian flow, if the flow rate at the inlet is kept constant, the velocity distribution is independent of the viscosity and the outlet pressure.

Unlike velocity, the pressure distribution is affected by viscosity. Figures 8 and 9 show the pressure development at the two ends of side channels. The pressure drops for horse blood within the side channels and the pressure in the main channel are higher than human blood. This shows that the pressure drop increases with increase in fluid viscosity. Note that the increase in pressure is similar to the increase in viscosity (4.7/3.5), as expressed by Eq (5).

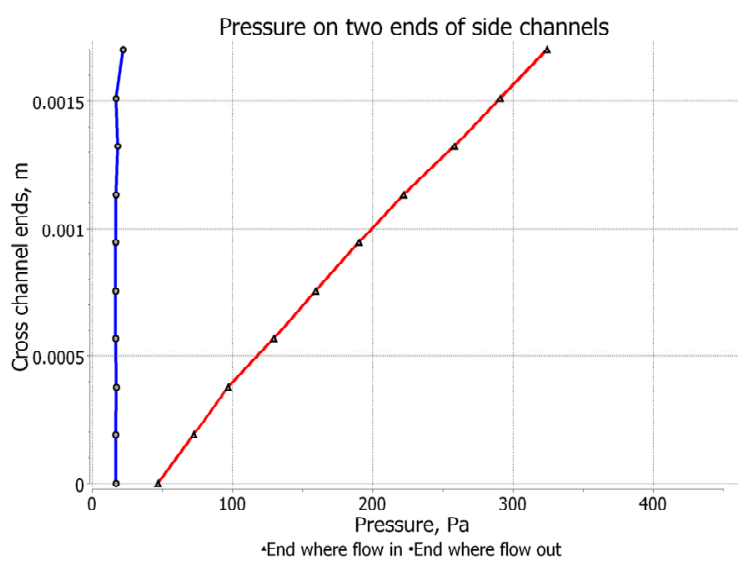

Figure 8 Flow pressure development at two ends of side channels, human blood flow

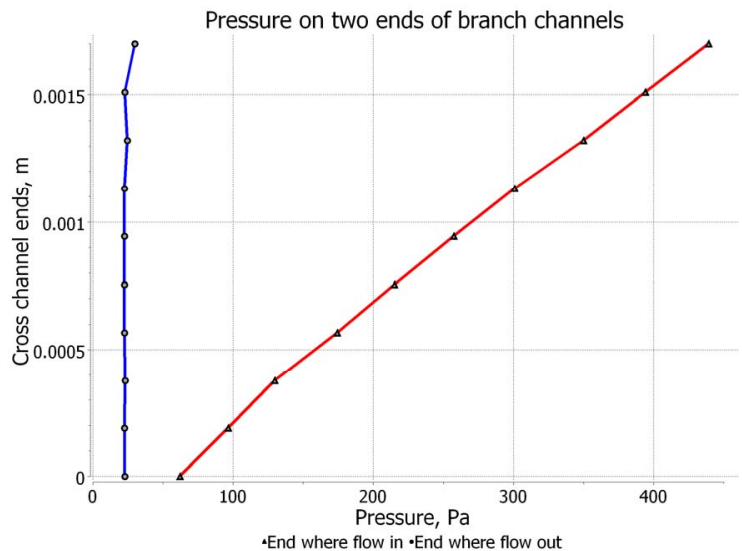

Figure 9 Pressure development at two ends of side channels, horse blood flow

The flow rate ratios at the bifurcations are shown in Table 1. All flow rate ratios are higher than the threshold value of 
$6: 1$ to $8: 1$ [12] required for pure plasma separation from the bulk flow. Thus, numerically, this biochip can theoretically separate plasma by nearly $100 \%$ from the bulk blood flow. For easy of comparison, the flow rate ratio modelled by nonNewtonian blood flow is also presented in Table 1.

Table 1 Flow rate ratios at bifurcations for both cases (No 1 refers the most upstream bifurcation)

\begin{tabular}{cccc}
\hline \multirow{2}{*}{$\begin{array}{c}\text { Bifurcation } \\
\text { number }\end{array}$} & $\begin{array}{c}\text { Newtonian } \\
\text { flow }\end{array}$ & & $\begin{array}{c}\text { Non-Newtonian } \\
\text { flow }\end{array}$ \\
\cline { 2 - 2 } & Human/horse & Human blood \\
\hline 1 & 25.89 & & 14.02 \\
3 & 27.06 & & 14.44 \\
4 & 28.95 & & 15.19 \\
5 & 30.02 & & 15.44 \\
6 & 33.12 & 16.75 \\
7 & 35.12 & 17.55 \\
8 & 37.65 & 18.49 \\
9 & 41.62 & 20.16 \\
10 & 45.73 & 21.81 \\
11 & 52.63 & 24.84 \\
12 & 62.51 & 29.05 \\
13 & 74.94 & 34.63 \\
14 & 94.47 & 43.52 \\
15 & 128.7 & 59.54 \\
\hline
\end{tabular}

\subsection{Modelling biofluids using non-Newtonian flow}

\subsubsection{Comparison of non-Newtonian and Newtonian flows}

Figures 10 and 11 present velocity profiles of the main and side channels for non-Newtonian flow. Comparing these to the results obtained for Newtonian fluids (Figures 5 and 6), the velocities within the side channels are largely increased and the velocity of the main channel is gradually reduced, as the fluid goes downstream. As a result, the flow rate ratio of the bifurcations has reduced (see Table 1). All the flow rate ratios at the bifurcations are higher than the separation threshold, indicating a successful separation.

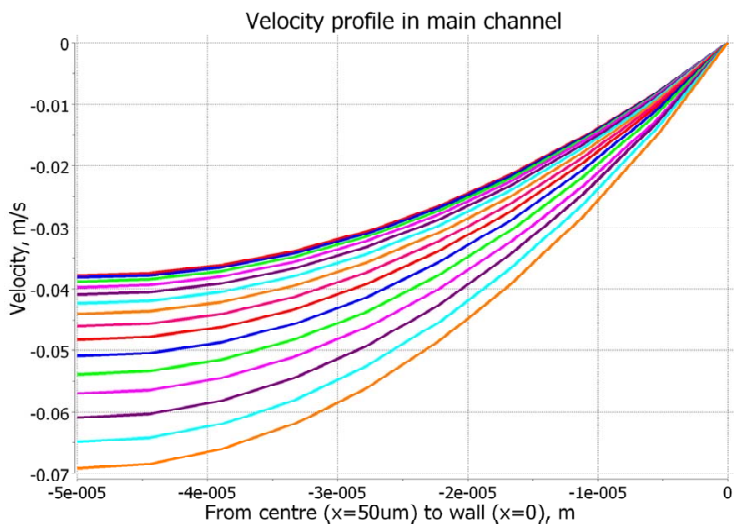

Figure 10 Flow velocity profile in the main channel

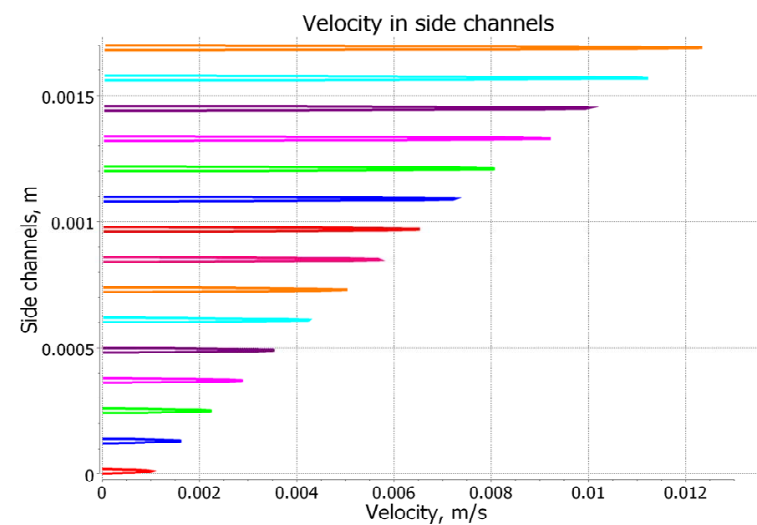

Figure 11 Flow velocity of side channels

Figure 12 shows the pressure development at the two ends of the side channels. The pressure expressed by the blue line (left) represents the pressure level at the outlet side of the side channels and the difference of two lines denotes the pressure drop over side channels. Both are largely decreased, as a result of the reduction of the fluid viscosity of the separated plasma from the bulk flow.

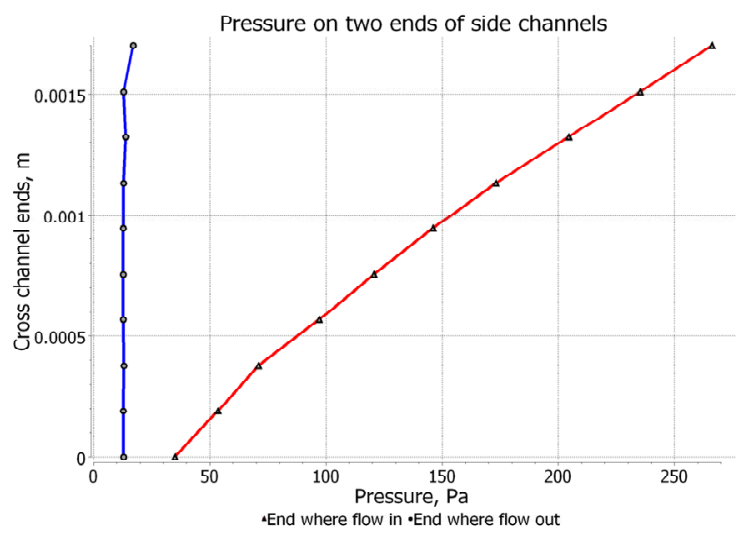

Figure 12 Pressure development at two ends of side channels, horse blood flow

In summary, comparing Newtonian and Non-Newtonian assumptions the flow rate of the side channels has largely increased and the flow rate of the main channel has gradually reduced as the fluid goes downstream. The pressures at the two sides of the side channels have reduced. Their difference, representing the pressure drop over the side channels, has also reduced. These lead to a microchannel biochip with low overall system pressure and fast and efficient plasma separation.

\subsubsection{Effect of flow rate/velocity on flow field}

To investigate the effect of the flow velocity on flow field, three cases with different velocities (flow rates), i.e. $10 \mathrm{~mm} / \mathrm{s}$ $(72 \mu \mathrm{l} / \mathrm{h}), 50 \mathrm{~mm} / \mathrm{s}(360 \mu \mathrm{l} / \mathrm{h})$ and $200 \mathrm{~mm} / \mathrm{s}(1.44 \mathrm{ml} / \mathrm{h})$, are simulated. Figure 13 shows the shear strain rate in the cases of $10 \mathrm{~mm} / \mathrm{s}$ and $200 \mathrm{~mm} / \mathrm{s}$ of input velocities. The distribution patterns are similar between the two cases. The rate of change in shear strain is similar as the input velocities. The $200 \mathrm{~mm} / \mathrm{s}$ case experiences some 20 times of shear strain rate as the 
$10 \mathrm{~mm} / \mathrm{s}$ case in the region close to the wall. As blood has shear-thinning material behaviour, viscosity in the $200 \mathrm{~mm} / \mathrm{s}$ is lower than the $10 \mathrm{~mm} / \mathrm{s}$ case (see Eq. (6)).

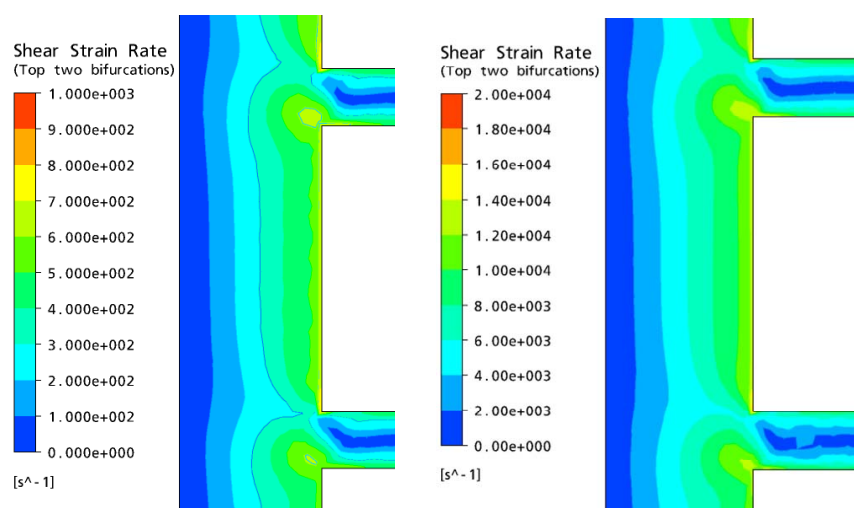

Figure 13 Shear strain rate at the top two bifurcations in the cases of $10 \mathrm{~mm} / \mathrm{s}$ (left) and $200 \mathrm{~mm} / \mathrm{s}$ (right) of input velocities

In microchannels, the shear strain rate is relevant to flow velocity. Flow velocity thus has an effect on the viscosity and this further affects the flow field. Table 2 shows maximum velocities at odd numbered bifurcations in the main and side channels. By defining the $10 \mathrm{~mm} / \mathrm{s}$ case as the reference case, the rates of increase in the maximum flow velocity by the other two cases can be obtained and are show in Table 3 .

Table 2 Maximum velocities of three input velocity cases at odd numbered bifurcations in the main and side channels

\begin{tabular}{ccccccc}
\hline \multirow{2}{*}{ No } & \multicolumn{3}{c}{ Main channel $(\mathrm{mm} / \mathrm{s})$} & \multicolumn{3}{c}{ Side channels $(\mathrm{mm} / \mathrm{s})$} \\
\cline { 2 - 7 } & $10 \mathrm{~mm} / \mathrm{s}$ & $50 \mathrm{~mm} / \mathrm{s}$ & $200 \mathrm{~mm} / \mathrm{s}$ & $10 \mathrm{~mm} / \mathrm{s}$ & $50 \mathrm{~mm} / \mathrm{s}$ & $200 \mathrm{~mm} / \mathrm{s}$ \\
\hline 1 & 13.64 & 69.17 & 277.9 & 2.595 & 12.34 & 47.98 \\
3 & 11.87 & 60.85 & 245.5 & 2.161 & 10.01 & 38.99 \\
5 & 10.38 & 53.91 & 218.4 & 1.752 & 8.047 & 31.28 \\
7 & 9.203 & 48.34 & 196.5 & 1.386 & 6.536 & 25.76 \\
9 & 8.288 & 44.04 & 179.4 & 1.059 & 5.049 & 19.99 \\
11 & 7.619 & 40.90 & 166.9 & 0.757 & 3.520 & 14.04 \\
13 & 7.169 & 38.82 & 158.5 & 0.478 & 2.230 & 9.006 \\
15 & 6.952 & 37.83 & 154.3 & 0.209 & 1.009 & 4.143 \\
\hline
\end{tabular}

Table 3 Change rate of velocity based on $10 \mathrm{~mm} / \mathrm{s}$ case at odd numbered bifurcations in the main and side channels

\begin{tabular}{cccccc}
\hline \multirow{2}{*}{$\begin{array}{c}\text { Bifurcation } \\
\text { No }\end{array}$} & \multicolumn{2}{c}{ Main channel } & & \multicolumn{2}{c}{ Side channels } \\
\cline { 2 - 3 } \cline { 5 - 6 } & $50 \mathrm{~mm} / \mathrm{s}$ & $200 \mathrm{~mm} / \mathrm{s}$ & & $50 \mathrm{~mm} / \mathrm{s}$ & $200 \mathrm{~mm} / \mathrm{s}$ \\
\hline 1 & 5.071 & 20.37 & & 4.755 & 18.49 \\
3 & 5.126 & 20.68 & & 4.632 & 18.04 \\
5 & 5.194 & 21.04 & & 4.593 & 17.85 \\
7 & 5.253 & 21.35 & & 4.716 & 18.59 \\
9 & 5.314 & 21.65 & & 4.768 & 18.88 \\
11 & 5.368 & 21.91 & & 4.650 & 18.55 \\
13 & 5.415 & 22.11 & & 4.665 & 18.84 \\
15 & 5.442 & 22.20 & & 4.828 & 19.82 \\
\hline Average & 5.273 & 21.41 & & 4.701 & 18.63 \\
\hline
\end{tabular}

From Table 3, the velocity distribution patterns are similar for the three cases, illustrating similar rates of change of velocities at different bifurcations in both the main and side channels. There are minor difference between the rate of change of the channel velocity and the input velocity, reflecting a limited effect of inlet velocity on the flow rate ratio. The average rates of bifurcation and input velocities from the $10 \mathrm{~mm} / \mathrm{s}$ case to the $50 \mathrm{~mm} / \mathrm{s}$ case are $5.3: 5$ for the main channel and 4.7:5 for the side channels. These values are much larger than those from the $50 \mathrm{~mm} / \mathrm{s}$ to $200 \mathrm{~mm} / \mathrm{s}$ case, i.e. 4:4 for the main and side channels. This shows that the effect of shear strain rate on viscosity and the flow field is nonlinear in behaviour. When velocity is high enough, the effect of the increased velocity becomes very limited.

The directions of the flow velocity change are different between the main and side channels. Velocity increase rates in the main channel are slightly higher than the difference of the inlet velocity values. By contrast, the rate of increase in the side channels is slightly lower than the rate of incease in the inlet velocities. This is because the viscosity in the main channel decreases with an increase in velocity, leading to more distribution of the volumetric flow through the main channel. In the side channels, due to the constant value of viscosity of the separated plasma, the increase in flow velocity cannot affect the flow rate through viscosity. As the total flow rate is fixed, the increase in velocity in the main channel leads to a decrease of velocity in the side channels. As a result, increasing input velocity for a non-Newtonian flow can result in a slight increase of the flow rate ratio between the main and side channels.

\section{Conclusions}

(1) The effect of mechanical (geometrical) features of the microchannel biochip on biofluid behaviour has been investigated. These features, i.e. microchannel, constriction, bifurcation, bending channel (or perpendicularly cross channels) and channel length ratio between the main and side channels, have shown strong effects on the biofluid hydrodynamics, bulk flow field and the separation efficiency. Some system improvement is therefore possible for the control of the flow field and the enhancement of the separation efficiency.

(2) Biofluid behaviour in the biochip is modelled with two Newtonian and one non-Newtonian flows cases. The comparison of two Newtonian flows cases shows that modelling has resulted in identifying that the velocity distribution in a flow rate controlled system does not depend on viscosity values. The comparison of non-Newtonian and Newtonian flow cases shows that modelling with the nonNewtonian representation; the results show an increase in plasma separation and an overall reduction of system pressure.

(3) For a non-Newtonian flow, an increase in the input flow rate can lead to an increase in the shear strain rate within the microchannel system and therefore, a decrease in the viscosity. Due to the different fluid features between the main and side channels, i.e. high cell concentration flow in the main channel and high plasma concentration flow in the side channels, the decrease in the viscosity affects more on the flow in the main channel than the side channels. More fluid volume will travel through the main channel than the side 
channels. This leads to a slight increase in the flow rate ratio between the main and side channels.

(4) In terms of plasma/blood separation, numerically this biochip can separate plasma from the bulk blood flow. Consider the common behaviour among biofluids; this design may also be suitable to other separation purposes.

\section{Acknowledgments}

The authors gratefully acknowledge the financial support by the UK Engineering and Physical Sciences Research Council (EPSRC) in funding the Grand Challenge Project '3D-Mintegration' (www.3D-mintegration.com), referenced $\mathrm{EP} / \mathrm{C} 534212 / 1$. The authors would also like to acknowledge the financial support of the Scottish Funding Council through the SRDG programme SCIMPS (Scottish Consortium in Integrated MicroPhotonics Systems).

\section{References}

1. Hester, J. P., Kellogg, R. M., Mulzet, A. P., Kruger, V. R., McCredie, K. B., Freireich, E. J., "Principles of blood separation and component extraction in a disposable continuous-flow single-stage channel," Blood, Vol. 54 (1979), pp. 254-68.

2. Carlson, R., Gabel, C., Chan, S., Austin, R., "Self-sorting of white blood cells in a lattice," Phys. Rev. Lett. Vol. 79 (1997), pp. 2149-2152.

3. Wilding, P., Kricka, L. J., Cheng, J., Hvichia, G., Shoffner, M. A., Fortina, P., "Integrated cell isolation and polymerase chain reaction analysis using silicon microfilter chambers," Anal. Biochem. Vol. 257 (1998), pp. 95-100.

4. Mohamed, H., Turner, J. N., Caggana, M., "Biochip for separating fetal cells from maternal circulation," Journal of Chromatography A, Vol. 1162 (2007), pp. 187-192.

5. Rainer, J., Roger, S., Carlo, E., "Microfluidic depletion of red blood cells from whole blood in high-aspect-ratio microchannels," Microfluid Nanofluid, Vol. 3 (2007), pp. 47-53.

6. Layek, G. C., Midya, C., "Effect of constriction height on flow separation in a two-dimensional channel," Commun Nonlinear Sci Numer Simul, Vol.12 (2007), pp.745-759.

7. Golia, C. and Evans, N. A., "Flow separation through annular constrictions in tubes," Experimental Mechanics, Vol 13, No. 4 (1973), pp. 157-162.

8. Blattert, C., Jurischka, R., Tahhan, I., Schoth, A., Kerth, P., Menz, W., "Microfluidic blood/plasma separation unit based on microchannel bend structures," Proc of microtechnology in medicine and biology, $3^{\text {rd }}$ IEEE/EMBS Special Topic Conf, Honolulu, Hawaii, 12-15 May 2005, pp. 38-41.

9. Carlo, D. D., Edd, J. F., Irlmla, D., Tompkins, R. G., Toner, M., "Equilibrium separation and filtration of particles using differential inertial focusing," Anal. Chem. Vol. 80 (2008), pp. 2204-2211.

10. Pries, A. R., Secomb, T. W., Gaehtgens, P., Gross, J. F., "Blood flow in microvascular networks, experiments and simulation," Circ Res. Vol. 67 (1990), pp. 826-834.

11. Jafari, A., Mousavi, S. M., Kolari, P., "Numerical investigation of blood flow, Part I: in microvessel bifurcations," Commun Nonlinear Sci Numer Simul, Vol. 13 (2008), pp. 1615-1626.

12. Yang, S., Undar, A., Zahn, J. D., "A microfluidic device for continuous, real time blood plasma separation," Lab Chip, Vol. 6 (2006), pp. 871-880.

13. Kersaudy-Kerhoas, M., Kavanagh, D, Xue X., Patel M.K., Dhariwal R., Bailey C., Desmulliez M.P.Y., "Integrated biomedical device for flood preparation," Proc of ESTC conf, London, 1-4 September 2008, pp. 447-452.

14. Dzwinel, W., Boryczko, K. and Yuen, D. A., "Discreteparticle model of blood dynamics in capillary vessels," Journal of Colloid and Interface Science, 258 (2003), pp. 163-173.

15. Korin, N., Bransky, A. and Dinnar, U., "Theoretical model and experimental study of red blood cell (RBC) deformation in microchannels," Journal of Biomechanics, Vol. 40 (2007), pp. 2088-2095.

16. Brown, B. H. et al., Medical Physics and Biomedical Engineering, Institute of Physics Publishing (Bristol, 1999)

17. Svanes, K., Zweifach, B. W., "Variations in small blood vessel hematocrit produced in hypothermic rats by microocclusion," Microvasc Res, Vol. 1 (1968), pp. 210-220.

18. Fung, Y. C. "Stochastic flow in capillary blood vessels," Microvasc. Res. Vol. 5 (1973), pp. 34-48.

19. Woodcock, J. P., "Physical properties of blood and their influence on blood-flow measurement," Rep. Prog. Phys. Vol. 39 (1976), pp. 65-127.

20. Kersaudy-Kerhoas, M., Dhariwal, R., Desmulliez, M.P.Y. and Jouvet, L., "Hydrodynamic blood plasma separation in microfluidic channels," Microfluid Nanofluid, DOI 10.1007/s10404-009-0450-5.

21. Dandy, D.S. and Dwyer, H. A., "A sphere in shear flow at finite Reynolds number: effect of shear on particle lift, drag, and heat transfer," J Fluid Mech, Vol. 216 (1990), pp. 381-410.

22. Saffman, P. G., "The lift on a small sphere in a slow shear flow," J Fluid Mech, Vol. 22 (1965), pp 385-400.

23. Dintenfass, L., Liao, F., "Plasma and blood viscosities, and aggregation of red cells in racehorses," Clin. Phys. Physiol. Meas., Vol.3 (1982), pp.293-301.

24. Waite, L., Fine, J. F., Applied Biofluid Mechanics. 1st ed. McGraw-Hill Professional, (New York 2007), pp. 30.

25. Leuprecht, A., Perktold, K., "Computer simulation of nonnewtonian effects on blood flow in large arteries," Comput. Methods Biomech. Biomed. Eng., Vol. 4 (2001), pp. 149-163.

26. Abraham, F., Behr, M., Heinkenschloss, M., "Shape optimization in steady blood flow: A numerical study of non-Newtonian effects," Comput. Methods Biomech. Biomed. Eng., Vol. 8, No. 2 (2005), pp. 127-137. 